\title{
Xanthogranulomatous Pyelonephritis in a Surgically Complicated Patient: A Case Report
}

\author{
Jooeun Kwon ${ }^{1}$, John N. Greene ${ }^{2}$ \\ ${ }^{1}$ University of South Florida Morsani College of Medicine, Tampa, FL, USA; \\ ${ }^{2} \mathrm{H}$. Lee Moffitt Cancer Center and Research Institute, Tampa, FL, USA
}

\begin{abstract}
Xanthogranulomatous pyelonephritis is an uncommon destructive process that is associated with urinary tract infection and/or obstruction. We present the case of a 63 year old female who had an unusual case of xanthogranulomatous pyelonephritis due to Streptococcus intermedius in the left kidney. CT scan revealed a chronic obstructive pyelonephritis with associated staghorn calculi, and renal histopathology confirmed xanthogranulomatous pyelonephritis with foamy macrophages. She was a surgically complicated patient, who underwent two ventral hernia repairs along with her left nephrectomy. She ultimately had a complete recovery after surgical treatment and antibiotics. J Microbiol Infect Dis 2019; 9(2):90-94.
\end{abstract}

Keywords: Xanthogranulomatous pyelonephritis, Streptococcus intermedius, case report

\section{INTRODUCTION}

Xanthogranulomatous pyelonephritis (XGP) is a rare and unusual form of chronic pyelonephritis or recurrent bacterial urinary tract infections. In the acute phase, XGP is characterized by inflammatory infiltrates consisting of Xanthomatous histiocytes, multinucleated giant cells, lymphocytes, plasma cells, and lipid laden macrophages. Inflammatory processes of XGP can lead to parenchymal destruction with affected areas in kidneys replaced by granulomatous tissues with lipid laden macrophages [1]. CT is the preferred diagnostic tool for XGP, but can be misdiagnosed as renal cell carcinoma in the cases of focal XGP [2,3]. XGP often presents in middle-aged women, most often unilaterally, with nonspecific symptoms that include fever, flank or abdominal pain, weight loss, gross hematuria, and fatigue [3]. We describe the first case of XGP associated with Streptococcus intermedius in a patient with a history of recurrent urinary tract infections with a complex surgical history.

\section{CASE}

A 63 year old female with a long history of recurrent urinary tract infections, kidney stones, and intra-abdominal infections, presented in February for consultation for a complex renal cyst and staghorn calculi, and possible left renal mass. The patient had seen a local urologist who advised left nephrectomy due to recurrent infections, large stone burden, and poorly functioning kidney; she deferred care due to financial reasons. She had no prior history of kidney stone removal surgery.

The patient has history of colonic obstruction resulting in colostomy in 2016, with subsequent takedown of colostomy in 2017 as well as an appendectomy. She subsequently developed a large ventral incisional hernia on the left side of the abdomen $(20 \times 15 \mathrm{~cm})$ following the colectomy, and a smaller hernia at the site of the appendectomy scar $(8 \times 4 \mathrm{~cm})$.

On physical examination, she was afebrile, and in no acute distress. Blood pressure, heart rate, and respiratory rate were normal. There was no flank pain or abdominal tenderness. A large ventral hernia and well-healed midline incision from prior surgery was noted. She denies recent urinary tract infection in the last six months and denies symptoms fever, dysuria, hematuria, urethral discharge, flank pain, chills, or shortness of breath.

Correspondence: Dr. John N. Greene, H. Lee Moffitt Cancer Center and Research Institute, 12902 Magnolia Drive, FOB-3,

Tampa, FL, USA

E-mail: John.Greene@moffitt.org

Received: 17 January 2019 Accepted: 28 April 2019

Copyright (C JMID / Journal of Microbiology and Infectious Diseases 2019, All rights reserved 
The patient's $\mathrm{CT}$ of the abdomen and pelvis during initial presentation in February 2018 showed chronic and obstructive xanthogranulomatous pyelonephritis with associated staghorn calculi (Figure 1). The mass appeared stable without definitive enhancement compared to her most recent images from November 2017. Her lab work upon admission for surgery in June 2018 showed WBC 20.19 $\mathrm{k} / \mathrm{uL}$, creatinine $1.0 \mathrm{mg} / \mathrm{dL}$, BUN $16 \mathrm{mg} / \mathrm{dL}$, and low estimated GFR of $56 \mathrm{~mL} / \mathrm{min} / 1.73 \mathrm{~m}^{2}$.

She underwent an exploratory laparotomy, lysis of adhesions, left radical nephrectomy, and ventral hernia repair with mesh. She was given cefazolin perioperatively for prophylaxis and continued on piperacillin-tazobactam after the procedure. A midline incision was made next to her prior incision, and she underwent lysis of her small and large bowel to further extend the excision and access the retroperitoneal space. Dissection of the superior pole of her kidney was difficult due to prior infections and abscesses, and frank pus was identified in an abscess cavity, which was sent for culture. She had a partial adrenalectomy as her left adrenal gland was plastered to the superior aspect of her kidney. The operative field required additional washout and irrigation with sterile water due to significant infection around the nephrectomy, but there was no evidence of entry into her pancreas.
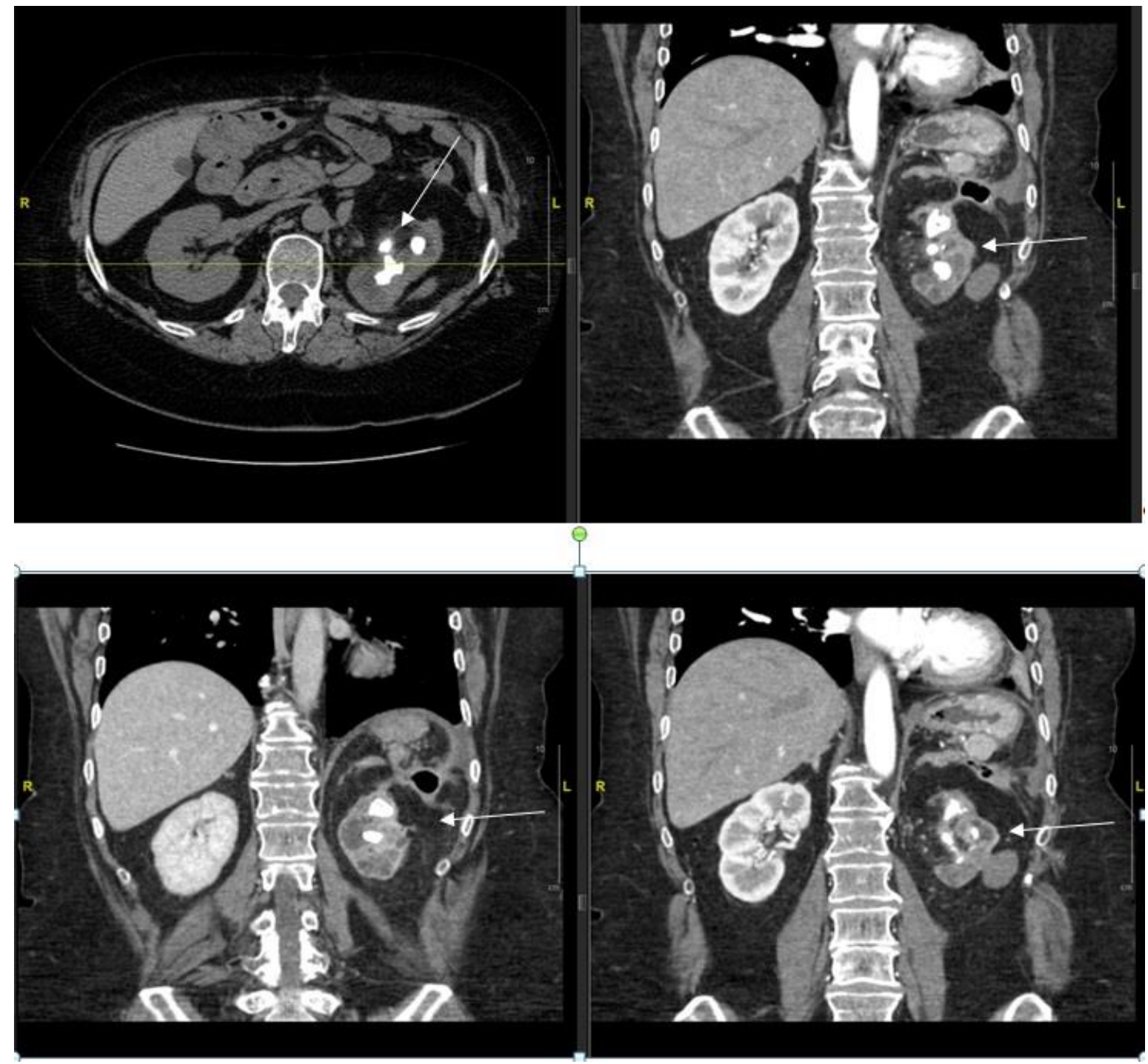

Figure 1. CT image is showing Chronic Obstructive Pyelonephritis with Staghorn Calculi. 
Following the nephrectomy, the patient then underwent repair of her two ventral hernias in which both hernia sacs were excised and sent to pathology. She then underwent a complex abdominal wall closure with components release separation over her left external oblique aponeurosis due to high tension upon attempting initial closure. A large onlay Marlex mesh was placed after closure of external oblique release.

The patient's urine culture was negative, but abscess culture grew Streptococcus intermedius which showed susceptibility to ampicillin, clindamycin, ceftriaxone, erythromycin, cefepime, levofloxacin, moxifloxacin, penicillin, and vancomycin. Histopathology of the resected kidney demonstrated xanthogranulomatous pyelonephritis with foamy macrophages, and pathology was negative for malignancy.

Her recovery was complicated by a fluid collection at the nephrectomy site, which required interventional radiology drain placement four days post-surgery. She had a retroperitoneal abscess with possible bowel leak/perforation after drain placement. Repeat CT of abdomen and pelvis six days post-surgery showed possible bowel injury and loculations. Subsequent CT of abdomen and pelvis eight days post-surgery showed her second abscess amenable to drainage. Her WBC continued to decrease to $11 \mathrm{k} / \mathrm{uL}$. She was continued on piperacillin-tazobactam and fluconazole as an inpatient. During follow up a month after surgery, her final CT of the abdomen and pelvis showed near complete resolution of collection in the left renal bed, and the patient reported minimal to no drain output. She completed a five-week course of ceftriaxone IV and fluconazole peroral after discharge and had a complete recovery with no further evidence of infection.

\section{DISCUSSION}

XGP is a rare but destructive type of chronic pyelonephritis or recurrent bacterial urinary tract infection that can occur in the setting of chronic obstruction from infected renal stones with an altered immune response. Xanthogranulomatous infections have been reported to affect various parts of the body but are most commonly seen in the kidney. In the acute phase, XGP is characterized by infiltration of inflammatory cells, and over time, XGP can lead to destruction of renal parenchyma through capillary proliferation, collagen formation, hemorrhage, and replacement by foamy macrophages [4].

$\mathrm{CT}$ is the preferred imaging modality as it has several advantages. CT can show replacement of renal tissue, the extent of the lesion, and clearly show renal stones, which is important in determining whether an obstruction contributing to XGP was malignant [3]. Final diagnosis of XGP is confirmed by pathology. Gross pathology often reveals enlarged kidney with inflammatory process and necrosis, often associated with renal stones or staghorn calculi within the mass. Inflammation can extend into surrounding structures and can lead to fistula formation [5]. Microscopic examination often reveals a center of necrosis and inflammatory infiltrate, surrounded by granulation tissue and vascularization, giant cells, and cholesterol clefts [4].

XGP is commonly associated with complete destruction of the kidney, so nephrectomy and surgical intervention of involved tissues is the mainstay of treatment [6]. Partial nephrectomy may be indicated in patients with a localized focal form of XGP or bilateral kidney involvement [7]. Our patient underwent a radical left nephrectomy as her XGP was diffuse, and there was a large stone burden with significant infection surrounding the kidney.

Due to prior surgeries and expected adhesions and inflammation, our patient had higher risk of surgical complications. Our patient had an exploratory laparotomy as she had to undergo two ventral hernia repairs and a nephrectomy. Laparoscopic ventral hernia repairs are associated with fewer superficial incision surgical site infections than the open technique [8]. However, given our patient's prior surgical history with expected adhesions and inflammation, a laparoscopic approach may have rendered the preperitoneal space inaccessible or made the surgery more challenging.

There are conflicting reports on the risk of infection in hernia repairs with and without a mesh. Mesh reinforcement can prevent hernia recurrence but is also associated with increased 
surgical site infection [9]. The ideal location of mesh placement is also debated. Ventral hernia repair with onlay mesh placement (over fascia) is technically less challenging than sublay placement (between rectus muscles and rectus sheath). Although placement of mesh over fascia has been associated with higher rate of wound infections based on existing studies, newer case-matched studies showed that onlay mesh placement is a safe alternative in low-risk patients [10]. In patients with large ventral hernias, advanced techniques such as component separation before mesh reinforcement are preferred, as was done in our patient [11]. While open hernia repairs and mesh placement may be associated with post-surgical infections, the copious irrigation during nephrectomy, the location of infectious drainage, and the use of antibiotics may precluded the patient from having further complications.

The most common pathogens isolated with XGP are Proteus mirabilis and Escherichia coli, although other organisms have been isolated, as listed in Table 1 [12]. S. intermedius was isolated from the abscess culture in this case. $S$. intermedius is a member of the Streptococcus anginosus group (also known as Streptococcus milleri group), which are gram positive, catalase negative cocci that are part of the normal oral and gastrointestinal flora. S. anginosus are known for their tendency to form abscesses, often as mixed infections with other Gl microorganisms. $S$. intermedius uniquely produces a cytolytic toxin called intermedilysin, which is a human specific virulence factor for deep seated abscess formation $[13,14]$.

Table 1. Pathogens Isolated in Patients with XGP (adapted from Kuo, CC et al (15))

\begin{tabular}{lc}
\hline Pathogens & $\%$ \\
\hline Escherichia coli & 45.7 \\
Proteus mirabilis & 20 \\
Bacteroides fragilis & 11.4 \\
Mixed pathogens & 22.8 \\
\hline
\end{tabular}

The patient was treated with ceftriaxone per susceptibility reports and fluconazole for five weeks after discharge, with no evidence of recurrent infections. Prophylactic fluconazole has been associated with reduced incidence of candidemia in high immunocompetent high-risk surgical patients [15]. The isolation of this unique pathogen in our patient may be associated with her colon obstruction and surgery. We report the first case of Streptococcus intermedius associated XGP with a successful outcome with a combination of surgical resection and antibiotic therapy.

\section{ACKNOWLEDGMENTS}

Declaration of Conflicting Interests: The authors declare that there is no conflict of interest regarding the publication of this paper.

Funding source: None declared.

\section{REFERENCES}

1. Cozzutto $\mathrm{C}$, Carbone A. The xanthogranulomatous process. Xanthogranulomatous inflammation. Pathol Res Pract 1988; 183(4): 395-402.

2. Hayes WS, Hartman DS, Sesterbenn IA. From the Archives of the AFIP. Xanthogranulomatous pyelonephritis. Radiographics 1991; 11(3): 485-498.

3. Zorzos I, Moutzouris V, Korakianitis G, Katsou G. Analysis of 39 cases of xanthogranulomatous pyelonephritis with emphasis on CT findings. Scand $\mathrm{J}$ Urol Nephrol 2003; 37(4): 342-347.

4. Bourm KS, Menias CO, Ali K, Alhalabi K, Elsayes $\mathrm{KM}$. Spectrum of Xanthogranulomatous Processes in the Abdomen and Pelvis: A Pictorial Review of Infectious, Inflammatory, and Proliferative Responses. Am J Roentg 2017; 208(3): 475-484.

5. Dawoud S, Solomon RJ, Eyerly-Webb SA, et al. Pyeloduodenal Fistula in Xanthogranulomatous Pyelonephritis: A Series of Two Cases. Perm J 2018; 22.

6. Malek RS, Elder JS. Xanthogranulomatous pyelonephritis: a critical analysis of 26 cases and of the literature. J Urol 1978; 119(5): 589-593.

7. Perez LM, Thrasher JB, Anderson EE. Successful management of bilateral xanthogranulomatous pyelonephritis by bilateral partial nephrectomy. J Urol 1993; 149(1): 100-102.

8. Sauerland S, Walgenbach M, Habermalz B, Seiler CM, Miserez M. Laparoscopic versus open surgical techniques for ventral or incisional hernia repair. Cochrane Database Syst Rev 2011(3): Cd007781.

9. Holihan JL, Hannon C, Goodenough C, et al. Ventral Hernia Repair: A Meta-Analysis of Randomized Controlled Trials. Surg Infect (Larchmt) 2017; 18(6): 647-658.

10. Haskins IN, Voeller GR, Stoikes NF, et al. Onlay with Adhesive Use Compared with Sublay Mesh Placement in Ventral Hernia Repair: Was Chevrel Right? An Americas Hernia Society Quality 
Collaborative Analysis. J Am Coll Surg 2017; 224(5): 962-970.

11. de Vries Reilingh TS, van Goor H, Rosman C, et al. Components separation technique" for the repair of large abdominal wall hernias. J Am Coll Surg 2003; 196(1): 32-37.

12. Addison B, Zargar H, Lilic N, Merrilees D, Rice M. Analysis of 35 cases of Xanthogranulomatous pyelonephritis. Anz Journal of Surgery 2014; 85(3): 150-153.

13. Nagamune $H$, Whiley RA, Goto $T$, et al. Distribution of the intermedilysin gene among the anginosus group streptococci and correlation between intermedilysin production and deep-seated infection with Streptococcus intermedius. J Clin Microbiol 2000; 38(1): 220-226.

14. Gossling J. Occurrence and pathogenicity of the Streptococcus milleri group. Rev Infect Dis 1988; 10(2): 257-285.

15. Ho KM, Lipman J, Dobb GJ, and Web SA. The use of prophylactic fluconazole in immunocompetent high-risk surgical patients: a meta-analysis. Crit Care 2005; 9(6): R710-17. 